\title{
Research on early warning and control of public opinion based on Digital twin
}

\author{
Wang Kai ${ }^{1}$, Gao Yang ${ }^{2}$, He Jie ${ }^{1}$ \\ ${ }^{1 .}$ Xijing University, Xi 'an , Shaanxi, China, 710123 \\ ${ }^{2}$ NavInfo Co.,Ltd. Xi 'an, Shaanxi, China, 710075 \\ *Corresponding email: 20180094@xijing.edu.cn

\begin{abstract}
This research focuses on how to use digital twinning technology to construct a simulation deduction system for public opinion warning in the face of public emergencies. It aims to improve the public opinion intelligent decision-making and response system, so as to enhance the ability of public opinion guidance, and to make a prediction, a decision and then a response in time on different aspects, like possibility of the public opinion, its size and time, even its development trend.
\end{abstract}

Keywords: public opinion warning, digital twinning, simulation deduction, intelligent decision-making

\section{基于数字孪生的舆情预警与管控研究}

王恺 ${ }^{1 *}$ 、高扬 ${ }^{2}$ 、何婕 ${ }^{1}$

1. 西京学院, 中国, 陕西, 西安, 710123

2. 西安四维图新信息技术有限公司, 中国，陕西，西安，710075

*通讯作者. 邮箱: 20180094@xijing. edu. cn

\section{摘要}

面向突发公共事件, 研究如何利用数字孪生技术, 构建與情预警仿真推演系统, 完善舆情智能决策及应对机制, 提升與情引导能力, 实时对舆情是否出现、出现时间、规模大小、发展走势、與情研判等方面的进行预判、决 策和应对。

关键词: 舆情预警; 数字孪生; 仿真推演; 智能决策

\section{1. 引言}

舆情管控是企业或政府正确把握自己的名誉的 重要环节, 是提升城市治理能力的重要内容。在以大 数据、云计算、移动互联网技术为代表的智能时代, 舆情数据呈现出突发性、爆炸性、不对称性等特点。 当前对舆情数据的研究主要是应用传媒学、系统动力 学、传染病模型等方法 ${ }^{[1-3]}$ 在传播模式、传播规律、传 播模型等方面进行理论探讨, ArcGIS、T00M 等也研发 了相应的舆情管控系统。但是, 在如何利用舆情数据 实现对舆情发展趋势的仿真推演, 进而实现智能的决 策分析方面, 还没太多研究。对舆情数据的智能处理 与仿真推演的研究尚处于起步阶段。针对舆情的预警 与管控, 本文在研究数字孪生技术的基础上, 对舆情
数据进行可视化的仿真分析、推演预判和智能决策, 构建舆情预警仿真推演系统模型, 并通过实际案例进 行验证。

\section{2. 大数据时代舆情管控的特点}

互联网新媒体每天都产生和承载着海量社会舆 情信息，这些信息蕴含着社会大众的评价和观点，如 果管控不到位，极有可能引发恶劣的群体行为，甚至 引发影响极大的社会與情事件。当前，基于新媒介传 播的舆情预警管控行动是一项实时动态的系统工程, 需在统一的指挥部门领导下多部门协同完成, 涉及公 安、通信、社区等多部门。

目前，舆情预警及管控的主要特点包括以下四个 
方面:

一是與情数据采集难。对與情预警及管控而言, 社交媒体是主要的與情信息源, 其信息量大, 数据重 复多样, 想要准确、实时地采集到这些数据比较困难。 信息采集不全, 直接对社会與情预警及管控的后续数 据处理及数据分析产生了较大的影响。

二是與情数据挖掘分析难度大。社会與情不仅信 息量大, 而且结构形式非常复杂, 传统舆情分析工具 难以统一分析处理。现有社会與情预警及管控系统在 数据处理方面, 一般是将采集的数据经过简单整理后 直接进行人工经验判断, 或者借助统计学方法进行研 判 ${ }^{[4]}$ 。对人工智能、数字孪生等解决大量的非结构化 数据的技术研究不够。数据挖掘分析直接会影响后续 的决策和控制。

三是缺乏有效的與情预警推演研判机制。與情特 别是网络與情的爆发周期缩短、传播速度加快, 留给 舆情管理部门监控、预警、处理的时间大大减少, 很 多情况下还没来得及处理, 與情就快速蔓延、爆发甚 至已经对社会公众和秩序产生了实际的影响, 这也给 政府的社会治理工作带来了较大的压力。加之现有的 與情预警及管控系统缺乏科学系统的研判指标和方 法 $^{[4]}$, 从而使得研判结果不科学, 决策的有效性降低。

\section{3. 基于数字孪生的舆情预警仿真推演系统设} 计

全球最具权威的 IT 研究与顾问咨询机构 Gartner 在 2017-2019 年报告中连续将数字孪生列为 十大战略科技发展趋势之一。数字孪生技术基于丰富 的历史、实时数据和先进的算法模型实现对对象状态 和行为高保真度的数字化表征、模拟试验和预测 ${ }^{[5]}$ 。 数字孪生是源自工业界的概念, 随着 $5 \mathrm{G}$ 通讯、物联 网、云计算、大数据、人工智能等新一代信息技术的 发展和广泛应用, 数字孪生在理论层面和应用层面均 取得了快速发展, 逐渐延伸到智慧城市、智慧园区、 智慧交通、智慧传媒等应用领域。

與情管控的核心需求和数字孪生的技术在数据 分析和仿真推演方面的优势高度契合。基于数字孪生 的與情预警仿真推演系统是通过数字化的手段对與 情实体对象进行动态定位、监测、仿真、分析、推演 和预判。仿真数据是实时数据在数字环境中的孪生和 映射, 实时数据与仿真数据形成双向闭环, 互相影响 和制约。

基于数字孪生的舆情预警仿真推演系统可为與 情预警、动态监控、人员时空分布、人员类型分析、 與情研判、與情趋势预测、與情态势评估等提供基本 支撑, 并为舆情预警管控行为效果进行预测和分析。 从整体上来讲, 與情预警仿真推演系统可提供 3 大类 数据服务: 與情时空大数据、监管区域人员基础信息、 舆情预警管控推演专题信息。涉及的数据形式有: 地 图矢量数据、地图栅格数据、实景影像数据、文本图
表及网络流媒体数据等。舆情预警仿真推演系统将这 些数据统一管理, 通过與情数据采集模型、信息融合 模型、與情传播效果评估模型分析、处理和推演, 得 出人文地理信息和舆情影响空间分布、舆情影响人员 分析、與情传播过程分析、舆情管控效果分析和舆情 传播态势分析等专题信息, 并将相关信息进行可视化 演示, 从而使监管人员能够实时的了解與情演变整体 状态和发展趋势, 为與情智能决策与应对, 與情预警 管控效果评估等提供辅助决策。與情数据工程流程如 图 1 所示。

與情预警仿真推演系统框架可分为三个层次, 即 数据处理层、计算仿真层和推演决策层。如图 2 所示。

一是数据处理层, 主要通过动态感知来获取数据。 在物联网、互联网技术的支撑下，系统可以动态的从 传统媒体、网络媒体、各类传感器实时动态地获取與 情数据, 从而更全面、及时地实现对與情环境的状态 感知。

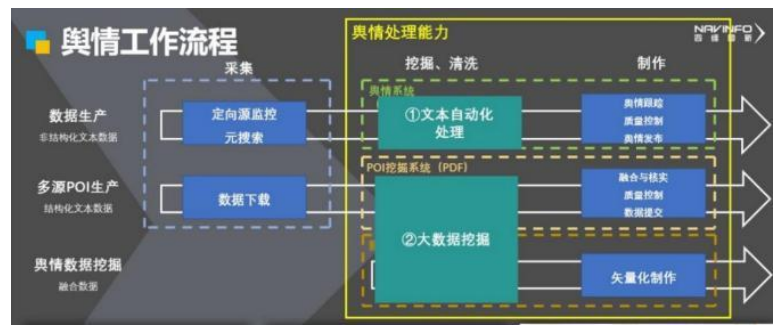

图 1 舆情数据工程流程

二是仿真推演层, 主要包含数据挖掘分析和仿真 推演两方面, 是为数字孪生上层智能决策应用提供技 术支撑的关键层次。一方面, 要对所获取数据的初级 模型进行深入挖掘, 建立更高层的知识模型, 即发现 一些规律、分布和关联, 获取出更高层语义的知识。 另一方面, 利用这些知识去做仿真演算, 从而能够为 上层应用实现通过现实的把握来预测未来。

三是智能决策层, 数字孪生的核心应用是预警和 决策。通过应用数字孪生, 可以在多个平行世界中通 过对基于历史数据的仿真推演来验证我们的策略和 决策是否合理，再进一步利用智能优化技术，不断迭 代改进策略和决策, 最后再通过控制系统来实施策略 和决策。

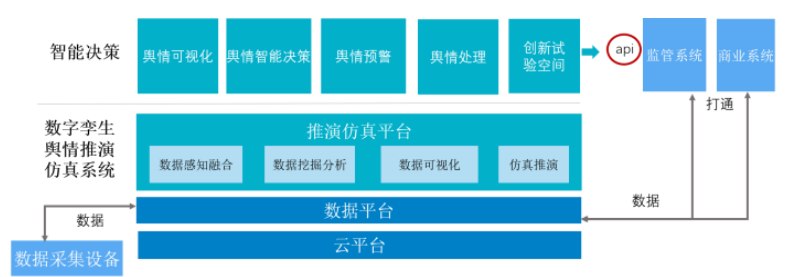

图 2 基于数字孪生的與情推演仿真系统

\section{4. 基于数字孪生的舆情推演仿真系统关键应 用}

基于数字孪生的與情预警仿真推演系统关键应 用具体表现为以下几个方面: 
第一，使用数字孪生技术可实现與情数据快速、 准确、有效地采集。以與情信息科学分类、充分获取 與情信息为基础, 使用数字孪生技术实现與情数据的 智能采集, 與情数据实时的反映舆情实际态势和趋势。 通过对與情数据进行清洗, 确保采集到各类舆情信息 全面、真实。政府部门和其他机构均可以根据自己的 权限从系统中得到相应的服务。

第二, 深度分析與情信息。对與情大数据进行数 据挖掘分析就是将與情中特定的事件进行智能识别、 对敏感的话题进行界定与分析、对有嫌疑的群体或个 人进行相应的行为分析。数字孪生应用于與情预警管 控, 是通过整合大数据采集技术、数据挖掘技术, 人 工智能和可视化技术, 对采集到的各类與情数据进行 采集、清洗、分类、聚类、分析和决策, 为各类用户 提供舆情监测、预警和管控等服务。通过可视化的分 析和决策结果, 为各用户部门提供分析依据, 有利于 提早做出與情预防、全面把握與情动态、及时做出與 情导控。基于数字孪生的與情预警仿真推演系统平台 实时记录着公众当时关注点与兴趣点、移动路径、性 格情绪、社会行为、社会关系链等特征。

第三, 实现各部门统一协调管理。舆情预警管控 的实践中, 相关部门众多, 他们在制订与实施社会與
情导控方案时, 就很难进行统一的沟通协调, 导致社 会舆情导控管理效果大打折扣, 从而增加社会與情事 件造成的影响和损失。通过数字孪生與情预警仿真推 演系统, 可以实现社会舆情预警管控相关的部门统筹 协作。通过数据平台产生的分析和决策数据可以实时 的分发给相关部门, 并实时生成可视化的决策方案, 实时联动各部门进行仿真推演, 从而使各部门的数据 格式统一、预警态势统一、决策部署统一, 通过线上 的协同实现线下的协调统一。

\section{5. 基于数字孪生舆情预警管控案例分析}

结合四维图新的时空大数据地理信息系统和数 据采集系统, 将基于数字孪生的與情预警仿真推演系 统在实际场景中进行了实验验证。通过数据采集、生 产、挖掘和制作建立與情预警与管控数据库。结合时 空大数据, 对采集处理的数据进行可视化处理, 实现 了與情数据来源、范围、发展趋势的可视化动态展示。 我们通过对不同区域的與情进行监控, 实时掌握该区 域交通设施、生活设施以及人员流动等情况, 通过與 情监控为地图数据的更新维护提供了情报信息。对與 情是否出现、出现时间、规模大小、发展走势等方面 进行了预判, 具体如图 3-4 所示。

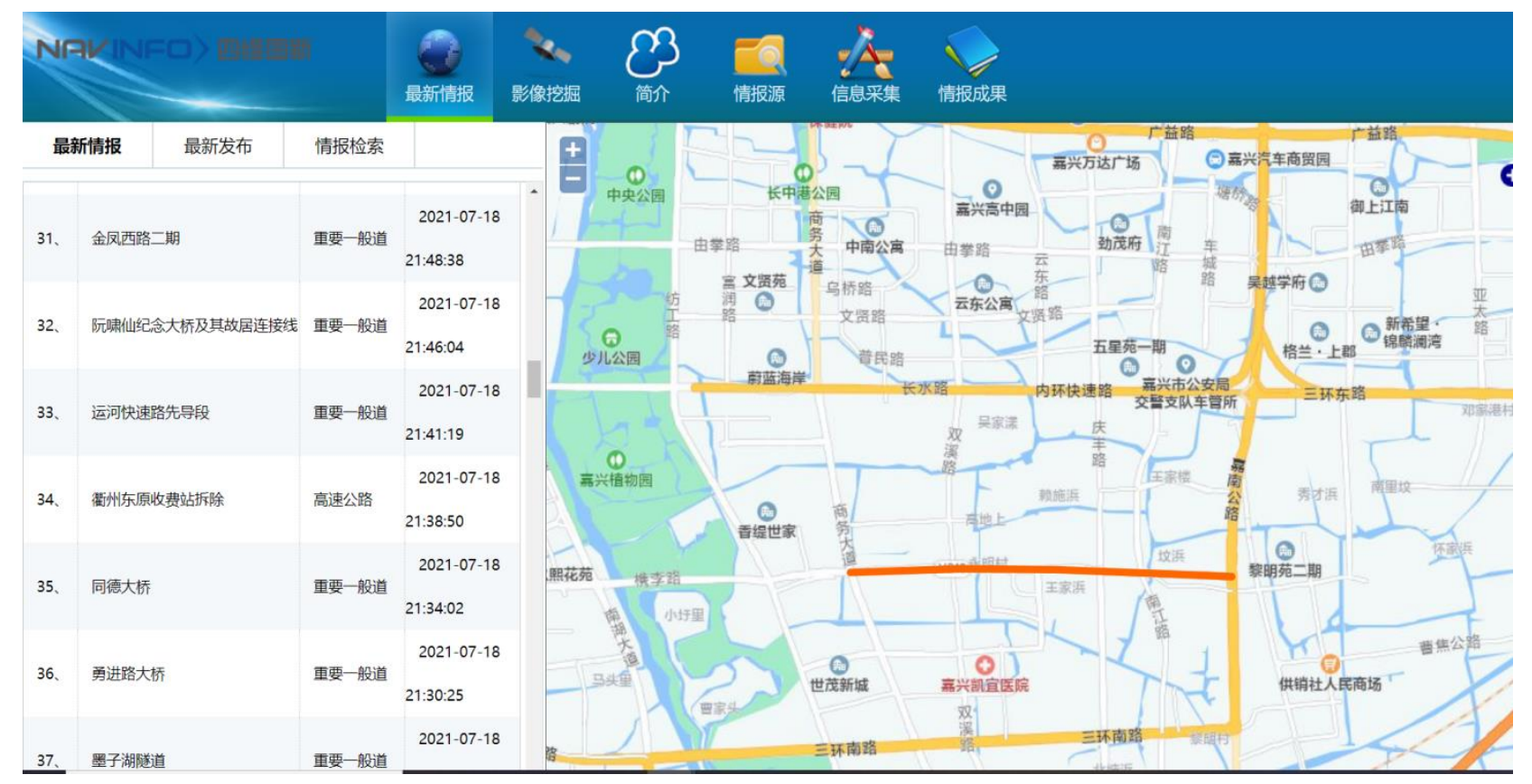

图 3 基于数字孪生的舆情数据采集可视化 -1 


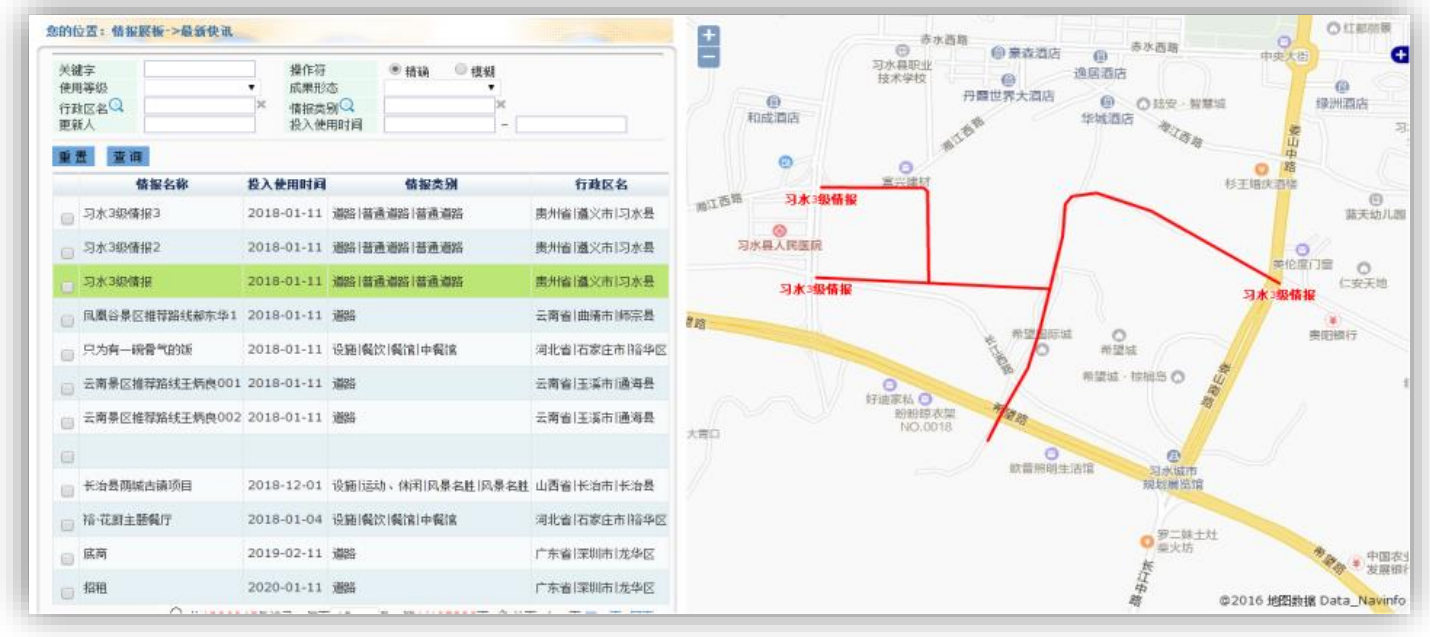

图 4 基于数字孪生的與情数据采集可视化 -2

\section{6. 结论}

本文对数字孪生技术在與情预警与管控中的应 用进行研究。围绕大数据时代舆情特点, 设计了基于 数字孪生的與情预警仿真推演系统, 对其关键应用进 行了阐述，并利用实际场景对系统进行了验证。面向 突发公共事件，要充分利用與情预警仿真推演系统， 不断提升对突发重大舆情信息的监测应对能力。

\section{REFERENCES}

[1] ZHANG SiLong, WANG Lancheng, LOU Guozhe, GAO Bin. Frontier evolution and development trend in studies on network public opinions in big data era. [J]. Chin J Med Libr Inf Sci, Vol.28 No.1:5055.

[2] Chen FuJi. SEIRS Research on Derivative Effects of Network Public Opinion Based on SEIRS Propagation Model[J]. Journal of Intelligence,

Vol.33 (2) :109-115.

[3] Dang XiaoChao. Research on Dissemination Model of Network Public Opinion Based on Fuzzy Cellular Automata[J]. Computer Engineering, Vol.40 (4) :209-213.

[4] Yang JunBin, Cao Min, Jia WangJing. Research on the Application of Big Data in Control and Precaution of Public Opinion[J]. Network Security Technology \& Application, 2018,09:68-69.

[5] Zhang TianYing, Ji Hang. Summary on Digital Twin [C]. SVTC 2019:74-79. 